\title{
Switching Fuzzy Guaranteed Cost Control for Nonlinear Networked Control Systems
}

\author{
Linqin Cai, ${ }^{1,2}$ Zhuo Yang, ${ }^{2}$ Jimin $Y u^{2}$ and Zhenhua Zhang ${ }^{2}$ \\ ${ }^{1}$ Key Laboratory of Industrial Internet of Things \& Networked Control, Ministry of Education, \\ Chongqing University of Posts and Telecommunications, Chongqing 400065, China \\ ${ }^{2}$ Research Center on Complex System Analysis and Control, Chongqing University of Posts and Telecommunications, \\ Chongqing 400065, China
}

Correspondence should be addressed to Linqin Cai; cailq@cqupt.edu.cn

Received 17 August 2013; Accepted 21 November 2013; Published 9 February 2014

Academic Editor: Bo-Chao Zheng

Copyright (C) 2014 Linqin Cai et al. This is an open access article distributed under the Creative Commons Attribution License, which permits unrestricted use, distribution, and reproduction in any medium, provided the original work is properly cited.

This paper deals with the problem of guaranteed cost control for a class of nonlinear networked control systems (NCSs) with time-varying delay. A guaranteed cost controller design method is proposed to achieve the desired control performance based on the switched T-S fuzzy model. The switching mechanism is introduced to handle the uncertainties of NCSs. Based on Lyapunov functional approach, some sufficient conditions for the existence of state feedback robust guaranteed cost controller are presented. Simulation results show that the proposed method is effective to guarantee system's global asymptotic stability and quality of service (QoS).

\section{Introduction}

As network technology advanced in the last decade, networked control system (NCS) has increasingly become a research focus. Considerable attention on the modeling and controller design of NCSs has been paid in [1-5]. There are many advantages to NCSs, such as reduced system wiring, facilitated system maintenance, and increased systems flexibility.

However, due to the insertion of communication channels, this brings many challenging problems such as networkinduced delay and data packet dropout. Regardless of the type of network used, these special issues degrade the system dynamic performance and are a source of potential instability. There are a number of design methods that have been proposed to deal with these problems. One of the most general methods is to model the NCS as a system with timevarying delays. So the stability of an NCS is equivalent to the stability of a system with time-varying delays [2]. Moreover, the sampling behavior has also an important impact on the design of the NCS controller because the states of the feedback controller are not continuous as a result of the existence of zeroth-order hold ( $\mathrm{ZOH})$. In [1], a model of NCS was provided considering network-induced delay and packet dropout in the transmission. In [3], an observer-based stabilizing controller was designed for the NCSs involving both random measurement and actuation delays. Robust controllers for uncertain NCSs were also obtained in $[4,5]$. However, how to analyze the stability of nonlinear NCSs has increasingly become a challenging topic. Some results about the stability of nonlinear NCSs were obtained in $[6,7]$. In [8], a stochastic optimal controller design for nonlinear NCSs with uncertain dynamics via neurodynamic programming was proposed. The closed-loop stability of the nonlinear NCSs was demonstrated by selecting neural network (NN) update laws. However, these methods often require some strict assumptions for a system model, so it is difficult for practical applications.

In the last few years, the fuzzy control is a useful approach to solve the control problems of nonlinear systems. The Takagi-Sugeno (T-S) fuzzy system proposed in [9] is widely applied to industrial control fields because of its simple structure with local dynamics. In the T-S fuzzy model, local dynamics in different state-space regions are represented by many linear models so that linear system theory can conveniently be employed to analyze the stability of overall closedloop system and to design the feedback controller. The typical design approaches are carried out based on fuzzy model 
via the so-called parallel distributed compensation (PDC) method [10]. Considering the time-delay characteristic of nonlinear systems, many results about the T-S fuzzy model with a time-delay term are obtained to deal with stability and stabilization problems of nonlinear systems with time delays $[2,11-13]$. The guaranteed cost control for a T-S fuzzy system with time delays was presented in $[2,11,12]$. In [13], robust control problem was studied for a class of large-scale NCSs, and the decentralized design was presented using T-S fuzzy approach.

Nevertheless, an inherent drawback remains since the number of fuzzy rules of a T-S model increases exponentially with the number of nonlinearities constituting the matched nonlinear system [14]. This makes fuzzy controller design and implementation difficult as the complexity of the nonlinear system to be controlled increases [15]. To outline the problem of rules explosion in fuzzy T-S modeling, some authors have proposed to combine the merit of switched systems with T-S ones to deal with nonlinear control problems [16-18]. To do so, partitioning the state space of a nonlinear system allows defining a switched nonlinear system. Then, inside each partition, a T-S model can be obtained. As stated in [18], the resulting switched T-S system inherits some essential features of hybrid systems and maintains all the information and knowledge representation capacity of fuzzy systems. However, few papers have studied stabilization issues of switched fuzzy systems based on switching Lyapunov functions [16] or quadratic approaches [17]. In [19], based on barrier Lyapunov functions (BLFs), a new control design for constrained nonlinear switched systems was investigated to achieve output tracking. By ensuring the boundedness of the BLFs in the closed loop, the proposed approaches can guarantee that all states in the switched systems do not violate the desired constraints and that all closed-loop signals are bounded. For many nonlinear systems, some of the premise variables of the corresponding T-S fuzzy models are measurable when they are modeled as T-S fuzzy models, while the parts with unmeasurable premise variables can be modeled as uncertainties. Thus, the overall systems can be described by T-S fuzzy parts with measurable premise variables and uncertainty parts. In [20], a switching stabilizing controller is designed for a nonlinear system with unknown parameters or unmeasurable premise variables. However, the uncertainties cannot be well handled. In [21], a switching fuzzy dynamic output feedback control scheme is proposed, where the switching mechanism is introduced to handle the unknown parameters.

In designing a controller for a real plant, it is invariably necessary to design a control system which not only is stable but also possesses a strong robust performance. One way to deal with this is the so-called guaranteed cost control approach [2]. This addresses the robust performance problem and has the advantage of providing an upper bound on a given performance index guaranteeing that system performance degradation incurred by uncertainty is less than this bound. For the NCSs, the quality of service (QoS) is one of the most important performance indexes. Therefore, it is vital to design a guaranteed cost controller such that the NCSs are stable as well as satisfactory with the required QoS.

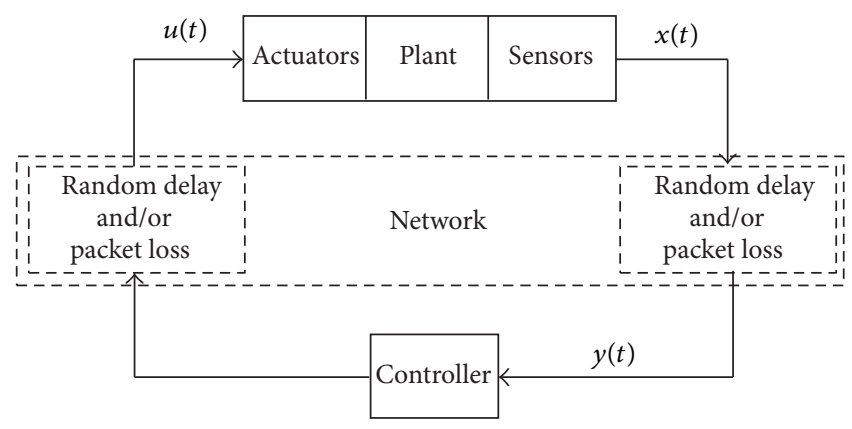

FIGURE 1: A general NCS.

In this paper, we aim at the problem of guaranteed cost control for a class of uncertain nonlinear NCSs with time delays. Considering the QoS of NCSs, we propose a guaranteed cost control scheme to achieve the desired control performance based on switched T-S fuzzy control method, where the switching mechanism is introduced to handle the uncertainties. Moreover, the sufficient condition for the existence of the robust guaranteed cost controller and the design method of the corresponding switching control law are obtained via Lyapunov functions. Comparing with [2, $11,12]$, the proposed switching fuzzy approach inherits some essential features of hybrid systems to deal with the uncertain nonlinear NCSs and avoids the inherent drawback of rules explosion in modeling a fuzzy T-S model. In addition, we consider the stabilization problem of the switched fuzzy T-S system with time delays in NCSs.

The innovations of this paper are as follows: (1) the guaranteed cost controller is proposed for nonlinear NCSs with time-varying delay to achieve the desired control performance based on the switched T-S fuzzy model with uncertain parameters, and (2) the sufficient condition for the robust guaranteed cost control law is presented to uncertain nonlinear NCSs.

The paper is organized as follows. The basic problem formulation of the nonlinear networked control system is given in Section 2. The switching fuzzy controller via state feedback is analyzed in Section 3. In Section 4, the sufficient stability conditions and guaranteed cost control law are discussed by Lyapunov functions. Section 5 provides simulation results to demonstrate the effectiveness of the proposed method. Finally, concluding remarks are given in Section 6.

\section{Problem Formulation}

A general NCS configuration is illustrated in Figure 1, which is composed of a controller and a remote system containing a physical plant, sensors, and actuators. The controller and the plant are physically located at different locations and are directly linked by a data network in order to perform remote closed-loop control.

In Figure 1, the nonlinear networked control system can be described as follows:

$$
\begin{aligned}
& \dot{x}(t)=f(x(t), u(t)), \\
& y(t)=g(x(t), u(t)),
\end{aligned}
$$


where $x(t) \in R^{n}$ is the state variation, $u(t) \in R^{m}$ is the control input, $y(t) \in R^{s}$ is the regulated output, $f(x, u)$ and $g(x, u)$ are the nonlinear functions. Currently, it has been proved that the T-S fuzzy models are universal approximations of many nonlinear dynamic systems. So we introduce a T-S fuzzy system to model a class of nonlinear NCSs. Without considering the disturbance input, we use the following T-S fuzzy model to approximate $f(x(t), u(t))$ and $g(x(t), u(t))$ :

$$
\begin{aligned}
& R_{i}: \operatorname{IF} \theta_{1}(t) \text { is } N_{i 1}, \ldots, \text { and } \theta_{g}(t) \text { is } N_{i g}, \\
& \operatorname{THEN}\left\{\begin{array}{l}
\dot{x}(t)=A_{i} x(t)+B_{i} u(t), \\
y(t)=C_{i} x(t),
\end{array}\right.
\end{aligned}
$$

where $i=1,2, \ldots, r$ is the index number of fuzzy rules, $\theta_{1}(t), \theta_{2}(t), \ldots, \theta_{g}(t)$ are the known premise variables, $N_{i k}$ is the fuzzy sets $(k=1,2, \ldots, g), x(t) \in R^{n}$ is the state vector, $y(t) \in R^{s}$ is the output vector, $u(t) \in R^{m}$ is the control input, $A_{i} \in R^{n \times n}$ is known system matrices, $B_{i} \in R^{n \times m}$ is the input matrix, and $C_{i} \in R^{n \times s}$ is the output matrix.

Before designing the controller, we make the following reasonable assumptions.

Assumption 1. The sensor is clock-driven. The controller and actuator are event-driven. The clocks among them are synchronized.

Assumption 2. Time-varying network-induced delay is less than one sampling period.

Assumption 3. The computational delay is negligible.

Assumption 4. The signal is single-packet transmission without packet drop.

Assuming the node sampling period is $T$, so we can obtain the discrete T-S fuzzy model of nonlinear networked control systems with time-varying delay:

$$
\begin{aligned}
& R_{i}: \operatorname{IF} \theta_{1}(k) \text { is } N_{i 1}, \ldots, \text { and } \theta_{g}(k) \text { is } N_{i g}, \\
& \text { THEN }\left\{\begin{array}{c}
x(k+1) \\
=\Phi_{i} x(k)+\Gamma_{i 0}\left(\tau_{k}\right) u(k) \\
+\Gamma_{i 1}\left(\tau_{k}\right) u(k-1), \\
y(k)=C_{i} x(k),
\end{array}\right.
\end{aligned}
$$

where $\Phi_{i}=e^{A_{i} T}, \Gamma_{i 0}\left(\tau_{k}\right)=\int_{0}^{T-\tau_{k}} e^{A_{i} T} d t \cdot B_{i}, \Gamma_{i 1}\left(\tau_{k}\right)=$ $\int_{T-\tau_{k}}^{T} e^{A_{i} T} d t \cdot B_{i}$, and $\tau_{k}$ is the network-induced delay, $i=$ $0,1,2, \ldots, r$.

Furthermore, (3) can be transformed into the following form with uncertain parameters:

$$
\begin{gathered}
x(k+1)=\Phi_{i} x(k)+\left(\Gamma_{i 0}+D_{i} F_{i}\left(\tau_{k}\right) E_{i}\right) u(k) \\
+\left(\Gamma_{i 1}-D_{i} F_{i}\left(\tau_{k}\right) E_{i}\right) u(k-1), \\
y(k)=C_{i} x(k),
\end{gathered}
$$

where $D_{i}, E_{i}$ are known constant matrices of appropriate dimensions and $F_{i}$ is an unknown matrix function satisfying

$$
\begin{gathered}
F^{T} F \leq I, \\
\Gamma_{i 0}\left(\tau_{k}\right)=\Gamma_{i 0}+D_{i} F_{i}\left(\tau_{k}\right) E_{i}, \\
\Gamma_{i 1}\left(\tau_{k}\right)=\Gamma_{i 1}-D_{i} F_{i}\left(\tau_{k}\right) E_{i} .
\end{gathered}
$$

For any given $x(k)$ and $u(k)$, by using a weighted-average defuzzifier, product inference, and singleton fuzzifier, the local models can be integrated into a global nonlinear model:

$$
\begin{gathered}
x(k+1)=\sum_{i=1}^{r} \mu_{i}(\theta(k))\left[\Phi_{i} x(k)+\Gamma_{i 0}\left(\tau_{k}\right) u(k)\right. \\
\left.+\Gamma_{i 1}\left(\tau_{k}\right) u(k-1)\right], \\
y(k)=C_{i} x(k),
\end{gathered}
$$

where

$$
\begin{aligned}
& \theta(k)=\left[\theta_{1}^{T}(k), \theta_{2}^{T}(k), \ldots, \theta_{g}^{T}(k)\right]^{T}, \\
& \mu_{i}(\theta(k))=\frac{\prod_{l=1}^{g} N_{i l}\left(q_{l}(k)\right)}{\sum_{i=1}^{r} \prod_{l=1}^{g} N_{i l}\left(\theta_{l}(k)\right)} .
\end{aligned}
$$

$N_{i l}\left(\theta_{l}(k)\right)$ is the grade of membership of $\theta_{l}(k)$ in the fuzzy set $N_{i l}$. Notice the following facts:

$$
\mu_{i}(\theta(k)) \geq 0, \quad \sum_{i=1}^{r} \mu_{i}(\theta(k))=1 .
$$

The guaranteed cost function associated with system (3) is given by

$$
J=\sum_{k=0}^{\infty}\left[x^{T}(k) Q x(k)+u_{\sigma}^{T}(k) R u_{\sigma}(k)\right] .
$$

Definition 5. Consider the uncertain system (3) and cost function (9). If there exists a control law $u_{\sigma}^{*}(k)$ and a positive scalar $J^{*}$ such that for all admissible uncertainties, the closedloop system is asymptotically stable and the value of the cost function (9) satisfies $J \leq J^{*}$, then $J^{*}$ is said to be a guaranteed cost and $u_{\sigma}^{*}(k)$ is said to be a guaranteed cost control law.

\section{Controller Design}

We assume that switched fuzzy controller is constituted with $N$ switching rules. The $\sigma$ th subfuzzy controller is

$$
\begin{aligned}
R_{i}^{\sigma}: & \operatorname{IF} \theta_{1}(t) \text { is } N_{i 1}, \ldots, \text { and } \theta_{g}(t) \text { is } N_{i g}, \\
& \operatorname{THEN} u_{\sigma}(k)=K_{\sigma i} x(k),
\end{aligned}
$$

where $\sigma=1,2, \ldots, N$ is a piecewise constant function representing the switching signal and $R_{i}^{\sigma}$ represents $i$ th fuzzy 
rule of the $\sigma$ th subfuzzy controller. The switching control law is constituted by the following fuzzy controller:

$$
\begin{aligned}
& u_{1}(k)=\sum_{i=1}^{r} \mu_{i}(\theta(k)) K_{1 i} x(k), \\
& u_{2}(k)=\sum_{i=1}^{r} \mu_{i}(\theta(k)) K_{2 i} x(k), \\
& \vdots \\
& u_{N}(k)=\sum_{i=1}^{r} \mu_{i}(\theta(k)) K_{N i} x(k) .
\end{aligned}
$$

When the controlled system is in $\sigma$ th switching subsystem, the global fuzzy equation is as follows

$$
\begin{aligned}
& x(k+1) \\
&= \sum_{i=1}^{r} \mu_{i}\left[\Phi_{i} x(k)+\Gamma_{i 0}\left(\tau_{k}\right) u(k)+\Gamma_{i 1}\left(\tau_{k}\right) u(k-1)\right] \\
&= \sum_{i=1}^{r} \mu_{i} \Phi_{i} x(k) \\
&+\sum_{i=1}^{r} \sum_{j=1}^{r} \mu_{i} \mu_{j}\left[\Gamma_{i 0}\left(\tau_{k}\right) K_{\sigma j} x(k)+\Gamma_{i 1}\left(\tau_{k}\right) K_{\sigma j} x(k-1)\right] \\
&=\sum_{i=1}^{r} \sum_{j=1}^{r} \mu_{i} \mu_{j} \quad \times\left[\Phi_{i} x(k)+\Gamma_{i 0}\left(\tau_{k}\right) K_{\sigma j} x(k)\right. \\
&\left.\quad+\Gamma_{i 1}\left(\tau_{k}\right) K_{\sigma j} x(k-1)\right] \\
&=\sum_{i=1}^{r} \sum_{j=1}^{r} \mu_{i} \mu_{j} \\
& \quad \times\left\{\left[\Phi_{i}+\Gamma_{i 0}\left(\tau_{k}\right) K_{\sigma j}\right] x(k)\right. \\
&\left.\quad+\Gamma_{i 1}\left(\tau_{k}\right) K_{\sigma j} x(k-1)\right\} \\
&=\sum_{i=1}^{r} \sum_{j=1}^{r} \mu_{i} \mu_{j}\left\{\left[\Phi_{i}+\left(\Gamma_{i 1}-D_{i} F_{i}\left(\tau_{k}\right) E_{i}\right) K_{\sigma j}\right] x(k)\right. \\
&\left.\quad+\left(\Gamma_{i 1}-D_{i} F_{i}\left(\tau_{k}\right) E_{i}\right) K_{\sigma j} x(k-1)\right\} .
\end{aligned}
$$

Let $\Omega_{1}, \Omega_{2}, \ldots, \Omega_{N}$ be a partition of the set $R^{n}$; then $\bigcup_{i=1}^{N} \Omega_{i}=$ $R^{n} \backslash\{0\}, \Omega_{i} \cap \Omega_{j}=\Phi, i=j$. The switching law that is determined by $\Omega_{1}, \Omega_{2}, \ldots$, and $\Omega_{N}$ is $\sigma=\sigma(x(t))=i$, when $x(t) \in \Omega_{i}$. This switching law can be completely described by the following function:

$$
v_{\sigma}(x(t))=\left\{\begin{array}{ll}
1, & x(t) \in \Omega_{\sigma}, \\
0, & x(t) \notin \Omega_{\sigma},
\end{array} \quad \sigma=1,2, \ldots, N .\right.
$$

Thus, we have

$$
\begin{aligned}
x(k+1)=\sum_{\sigma=1}^{N} \sum_{i=1}^{r} \sum_{j=1}^{r} v_{\sigma} \mu_{i} \mu_{j} & \\
\times & \left\{\left[\Phi_{i}+\left(\Gamma_{i 1}-D_{i} F_{i}\left(\tau_{k}\right) E_{i}\right) K_{\sigma j}\right] x(k)\right. \\
& \left.+\left(\Gamma_{i 1}-D_{i} F_{i}\left(\tau_{k}\right) E_{i}\right) K_{\sigma j} x(k-1)\right\},
\end{aligned}
$$

where $\tau_{k}$ is the network-induced delay.

\section{Sufficient Condition for \\ Guaranteed Cost Control}

Lemma 6 (see [22] (Schur complement)). For a given symmetric matrix,

$$
S=\left[\begin{array}{ll}
S_{11} & S_{12} \\
S_{21} & S_{22}
\end{array}\right] .
$$

Then, the following three conditions are mutually equivalent:

$$
\begin{aligned}
& \text { (1) } S<0, \\
& \text { (2) } S_{11}<0, S_{22}-S_{12}^{T} S_{11}^{-1} S_{12}<0 \text {, } \\
& \text { (3) } S_{22}<0, S_{11}-S_{12} S_{22}^{-1} S_{12}^{T}<0 .
\end{aligned}
$$

Lemma 7 (see [23]). Given matrices $Y, D, E$, and I of appropriate dimensions and with $Y$ and $I$ symmetrical and $I>0$, then

$$
Y+D F E+E^{T} F^{T} D^{T}<0
$$

for all $F$ satisfying $F^{T} F \leq I$, if and only if there exists some $\varepsilon>0$ such that

$$
Y+\varepsilon^{2} D D^{T}+\varepsilon^{-2} E^{T} E<0 .
$$

Theorem 8. Consider the uncertain nonlinear networked control systems (3) and the cost function (9). If there exist some constants $\lambda_{\sigma} \in[0,1], \sum_{\sigma=1}^{l} \lambda_{\sigma}=1$, a group of positive constants $\varepsilon_{\sigma}>0$, and positive definite matrices $X, Z, Y_{\sigma i}$, $\sigma=1, \ldots, l, i=1, \ldots, r$, such that the following matrix inequalities (18) hold:

$$
\sum_{\sigma=1}^{l} \lambda_{\sigma}\left[\begin{array}{ccccccc}
L & U & V & 0 & 0 & 0 & 0 \\
* & -2 X+2 Z & 0 & 2 X & \left(Y_{\sigma i}+Y_{\sigma j}\right)^{T} & \left(E_{i} Y_{\sigma j}\right)^{T} & \left(E_{j} Y_{\sigma i}\right)^{T} \\
* & * & -2 Z & 0 & 0 & -\left(E_{i} Y_{\sigma j}\right)^{T} & -\left(E_{j} Y_{\sigma i}\right)^{T} \\
* & * & * & -2 Q^{-1} & 0 & 0 & 0 \\
* & * & * & * & -2 R^{-1} & 0 & 0 \\
* & * & * & * & * & -\varepsilon_{\sigma} I & 0 \\
* & * & * & * & * & * & -\varepsilon_{\sigma} I
\end{array}\right]<0,
$$


where

$$
\begin{gathered}
X=P^{-1}, \quad Y_{\sigma i}=k_{\sigma i} X, \\
Z=X S X, \\
L=-2 X+\varepsilon_{\sigma}\left(D_{i} D_{i}^{T}+D_{j} D_{j}^{T}\right), \\
U=\Phi_{i}+\Phi_{j}+\Gamma_{i 0} Y_{\sigma j}+\Gamma_{j 0} Y_{\sigma i}, \\
V=\Gamma_{i 1} Y_{\sigma j}+\Gamma_{j 1} Y_{\sigma i},
\end{gathered}
$$

then close-loop system (14) with the guaranteed cost controller (11) and the switching law $\sigma=\sigma(x(k))$ is globally asymptotically stable. The guaranteed cost function (9) satisfies the following bound:

$$
J \leq x^{T}(0) P x(0)+x^{T}(-1) S x(-1) .
$$

Proof. For the networked control system (3), we lead the performance index as follows:

$$
\begin{aligned}
& J=\sum_{k=0}^{\infty}\left[x^{T}(k) Q x(k)+u_{\sigma}^{T}(k) R u_{\sigma}(k)\right] \\
&= \sum_{k=0}^{\infty}\left[\sum_{i=1}^{r} \sum_{j=1}^{r} \mu_{i} \mu_{j} x^{T}(k)\left(Q+K_{\sigma i}^{T} R K_{\sigma j}\right) x(k)\right] \\
&=\sum_{k=0}^{\infty}\left[\sum_{i=1}^{r} \mu_{i}^{2} x^{T}(k)\left(Q+K_{\sigma i}^{T} R K_{\sigma i}\right) x(k)\right. \\
&\left.+2 \sum_{i=1}^{r} \sum_{j>i}^{r} \mu_{i} \mu_{j} x^{T}(k)\left(Q+K_{\sigma i}^{T} R K_{\sigma j}\right) x(k)\right]
\end{aligned}
$$

$$
\begin{aligned}
=\sum_{k=0}^{\infty}\left[\sum_{i=1}^{r} \mu_{i}^{2} x^{T}(k)\left(Q+K_{\sigma i}^{T} R K_{\sigma i}\right) x(k)\right. \\
+2 \sum_{i=1}^{r} \sum_{j>i}^{r} \mu_{i} \mu_{j} x^{T}(k) \\
\left.\quad \times\left(Q+\frac{\left(K_{\sigma i}+K_{\sigma j}\right)^{T}}{2} R \frac{\left(K_{\sigma i}+K_{\sigma j}\right)}{2}\right) x(k)\right] .
\end{aligned}
$$

Consider the Lyapunov function as

$$
\begin{aligned}
V(x(k))= & x^{T}(k) \operatorname{Px}(k) \\
& +x^{T}(k-1) S x(k-1) .
\end{aligned}
$$

Let

$$
\begin{gathered}
\Theta=\left[\begin{array}{cc}
G_{\sigma i j}^{T} P G_{\sigma n m}-P+S & G_{\sigma i j}^{T} P H_{\sigma n m} \\
H_{\sigma i j}^{T} P G_{\sigma n m} & H_{\sigma i j}^{T} P H_{\sigma n m}-S
\end{array}\right], \\
G_{\sigma i j}=\Phi_{i}+\Gamma_{i 0} K_{\sigma j}+D_{i} F_{i}\left(\tau_{k}\right) E_{i} K_{\sigma j}, \\
\alpha_{\sigma i j}=G_{\sigma i j}+G_{\sigma j i}, \\
H_{\sigma i j}=\Gamma_{i 1} K_{\sigma j}-D_{i} F_{i}\left(\tau_{k}\right) E_{i} K_{\sigma j}, \\
\beta_{\sigma i j}=H_{\sigma i j}+H_{\sigma j i} .
\end{gathered}
$$

Along any trajectory of the closed-loop system (14), the forward difference of $V(k)$ is

$$
\begin{aligned}
& \Delta V(x(k))=V(x(k+1))-V(x(k)) \\
& =x^{T}(k+1) P x(k+1)+x^{T}(k) S x(k)-x^{T}(k) P x(k)-x^{T}(k-1) S x(k-1) \\
& =\sum_{\sigma=1}^{N} \sum_{i=1}^{r} \sum_{j=1}^{r} \sum_{n=1}^{r} \sum_{m=1}^{r} v_{\sigma} \mu_{i} \mu_{j} \mu_{n} \mu_{m}\left[\begin{array}{c}
x(k) \\
x(k-1)
\end{array}\right]^{T} \Theta\left[\begin{array}{c}
x(k) \\
x(k-1)
\end{array}\right] \\
& \leq \sum_{\sigma=1}^{N} v_{\sigma}\left\{\frac{1}{4} \sum_{i=1}^{r} \sum_{j=1}^{r} \mu_{i} \mu_{j}\left[\begin{array}{c}
x(k) \\
x(k-1)
\end{array}\right]^{T}\left[\begin{array}{cc}
\alpha_{\sigma i j}^{T} P \alpha_{\sigma i j}-4 P+4 S & \alpha_{\sigma i j}^{T} P \beta_{\sigma i j} \\
\beta_{\sigma i j}^{T} P \alpha_{\sigma i j} & \beta_{\sigma i j}^{T} P \beta_{\sigma i j}-4 S
\end{array}\right]\left[\begin{array}{c}
x(k) \\
x(k-1)
\end{array}\right]\right\} \\
& =\sum_{\sigma=1}^{N} v_{\sigma}\left\{\sum_{i=1}^{r} \mu_{i}^{2}\left[\begin{array}{c}
x(k) \\
x(k-1)
\end{array}\right]^{T}\left[\begin{array}{cc}
G_{\sigma i i}^{T} P G_{\sigma i i}-P+S & G_{\sigma i i}^{T} P H_{\sigma i i} \\
H_{\sigma i i}^{T} P G_{\sigma i i} & H_{\sigma i i}^{T} P H_{\sigma i i}-S
\end{array}\right]\left[\begin{array}{c}
x(k) \\
x(k-1)
\end{array}\right]\right. \\
& \left.+2 \sum_{i=1}^{r} \sum_{j>i}^{r} \mu_{i} \mu_{j}\left[\begin{array}{c}
x(k) \\
x(k-1)
\end{array}\right]^{T}\left[\begin{array}{cc}
{\left[\frac{\alpha_{\sigma i j}}{2}\right]^{T} P \frac{\alpha_{\sigma i j}}{2}-P+S} & {\left[\frac{\alpha_{\sigma i j}}{2}\right]^{T} P \frac{\beta_{\sigma i j}}{2}} \\
{\left[\frac{\beta_{\sigma i j}}{2}\right]^{T} P \frac{\alpha_{\sigma i j}}{2}} & {\left[\frac{\beta_{\sigma i j}}{2}\right]^{T} P \frac{\beta_{\sigma i j}}{2}-S}
\end{array}\right]\left[\begin{array}{c}
x(k) \\
x(k-1)
\end{array}\right]\right\} .
\end{aligned}
$$


Thus

$$
\begin{aligned}
& \Delta V(x(k))+x^{T}(k) Q x(k)+u_{\sigma}^{T}(k) R u_{\sigma}(k) \\
& \leq \sum_{\sigma=1}^{N} v_{\sigma}\left\{\sum_{i=1}^{r} \mu_{i}^{2}\left[\begin{array}{c}
x(k) \\
x(k-1)
\end{array}\right]^{T}\right. \\
& \times\left[\begin{array}{cc}
G_{\sigma i i}^{T} P G_{\sigma i i}-P+S & G_{\sigma i i}^{T} P H_{\sigma i i} \\
H_{\sigma i i}^{T} P G_{\sigma i i} & H_{\sigma i i}^{T} P H_{\sigma i i}-S
\end{array}\right]\left[\begin{array}{c}
x(k) \\
x(k-1)
\end{array}\right] \\
& +2 \sum_{i=1}^{r} \sum_{j>i}^{r} \mu_{i} \mu_{j}\left[\begin{array}{c}
x(k) \\
x(k-1)
\end{array}\right]^{T}\left[\begin{array}{cc}
{\left[\frac{\alpha_{\sigma i j}}{2}\right]^{T} P \frac{\alpha_{\sigma i j}}{2}-P+S} & {\left[\frac{\alpha_{\sigma i j}}{2}\right]^{T} P \frac{\beta_{\sigma i j}}{2}} \\
{\left[\frac{\beta_{\sigma i j}}{2}\right]^{T} P \frac{\alpha_{\sigma i j}}{2}} & {\left[\frac{\beta_{\sigma i j}}{2}\right]^{T} P \frac{\beta_{\sigma i j}}{2}-S}
\end{array}\right]\left[\begin{array}{c}
x(k) \\
x(k-1)
\end{array}\right] \\
& +\sum_{i=1}^{r} \mu_{i}^{2} x^{T}(k)\left(Q+K_{\sigma i}^{T} R K_{\sigma i}\right) x(k) \\
& \left.+2 \sum_{i=1}^{r} \sum_{j>i}^{r} \mu_{i} \mu_{j} x^{T}(k)\left(Q+\frac{\left(K_{\sigma i}+K_{\sigma j}\right)^{T}}{2} R \frac{\left(K_{\sigma i}+K_{\sigma j}\right)}{2}\right) x(k)\right\} \\
& =\sum_{\sigma=1}^{N} v_{\sigma}\left\{\sum_{i=1}^{r} \mu_{i}^{2}\left[\begin{array}{c}
x(k) \\
x(k-1)
\end{array}\right]^{T}\right. \\
& \times\left[\begin{array}{cc}
G_{\sigma i i}^{T} P G_{\sigma i i}-P+S+Q+K_{\sigma i}^{T} R K_{\sigma i} & G_{\sigma i i}^{T} P H_{\sigma i i} \\
H_{\sigma i i}^{T} P G_{\sigma i i} & H_{\sigma i i}^{T} P H_{\sigma i i}-S
\end{array}\right]\left[\begin{array}{c}
x(k) \\
x(k-1)
\end{array}\right] \\
& +2 \sum_{i=1}^{r} \sum_{j>i}^{r} \mu_{i} \mu_{j}\left[\begin{array}{c}
x(k) \\
x(k-1)
\end{array}\right]^{T} \\
& \times\left[\begin{array}{cc}
{\left[\frac{\alpha_{\sigma i j}}{2}\right]^{T} P \frac{\alpha_{\sigma i j}}{2}-P+S+Q+\frac{\left(K_{\sigma i}+K_{\sigma j}\right)^{T}}{2} R \frac{\left(K_{\sigma i}+K_{\sigma j}\right)}{2}} & {\left[\frac{\alpha_{\sigma i j}}{2}\right]^{T} P \frac{\beta_{\sigma i j}}{2}} \\
{\left[\frac{\beta_{\sigma i j}}{2}\right]^{T} P \frac{\alpha_{\sigma i j}}{2}} & {\left[\frac{\beta_{\sigma i j}}{2}\right]^{T} P \frac{\beta_{\sigma i j}}{2}-S}
\end{array}\right] \\
& \left.\times\left[\begin{array}{c}
x(k) \\
x(k-1)
\end{array}\right]\right\} \text {. }
\end{aligned}
$$

Thus, if the matrix inequalities (26) and (27) hold

$$
\begin{gathered}
{\left[\begin{array}{cc}
G_{\sigma i i}^{T} P G_{\sigma i i}-P+S+Q+K_{\sigma i}^{T} R K_{\sigma i} & G_{\sigma i i}^{T} P H_{\sigma i i} \\
H_{\sigma i i}^{T} P G_{\sigma i i} & H_{\sigma i i}^{T} P H_{\sigma i i}-S
\end{array}\right]<0, \quad 1 \leq i \leq r,} \\
{\left[\frac{\alpha_{\sigma i j}}{2}\right]^{T} P \frac{\alpha_{\sigma i j}}{2}-P+S+Q+\frac{\left(K_{\sigma i}+K_{\sigma j}\right)^{T}}{2} R \frac{\left(K_{\sigma i}+K_{\sigma j}\right)}{2}} \\
\left.\left[\frac{\beta_{\sigma i j}}{2}\right]^{T} P \frac{\alpha_{\sigma i j}}{2}\right]^{T} P \frac{\alpha_{\sigma i j}}{2} \\
\left.\left[\frac{\beta_{\sigma i j}}{2}\right]^{T} P \frac{\beta_{\sigma i j}}{2}-S\right]<0, \quad 1 \leq i<j \leq r,
\end{gathered}
$$


then, the following inequality can hold:

$$
\Delta V(x(k))+x^{T}(k) Q x(k)+u_{\sigma}^{T}(k) R u_{\sigma}(k)<0,
$$

and (26) is the special case of (27) when $i=j$. Thus, when (27) holds, the inequality (28) can hold. Consider

$$
\begin{aligned}
& \theta_{\sigma i j}=\left[\begin{array}{cc}
{\left[\frac{\alpha_{\sigma i j}}{2}\right]^{T} P \frac{\alpha_{\sigma i j}}{2}-P+S+Q+\frac{\left(K_{\sigma i}+K_{\sigma j}\right)^{T}}{2} R \frac{\left(K_{\sigma i}+K_{\sigma j}\right)}{2}} & {\left[\frac{\alpha_{\sigma i j}}{2}\right]^{T} P \frac{\beta_{\sigma i j}}{2}} \\
{\left[\frac{\beta_{\sigma i j}}{2}\right]^{T} P \frac{\alpha_{\sigma i j}}{2}} & \left.\left[\frac{\beta_{\sigma i j}}{2}\right]^{T} P \frac{\beta_{\sigma i j}}{2}-S\right]<0,
\end{array}\right]< \\
& 1 \leq i \leq j \leq r .
\end{aligned}
$$

Let

$$
\begin{array}{r}
\Omega_{\sigma i j}=-4 P+4 S+4 Q+\left(K_{\sigma i}+K_{\sigma j}\right)^{T} R\left(K_{\sigma i}+K_{\sigma j}\right), \\
\theta_{\sigma i j}=\left[\begin{array}{cc}
\Omega_{\sigma i j}+\alpha_{\sigma i i}^{T} P \alpha_{\sigma i j} & \alpha_{\sigma i i}^{T} P \beta_{\sigma i j} \\
\beta_{\sigma i i}^{T} P \alpha_{\sigma i j} & \beta_{\sigma i i}^{T} P \beta_{\sigma i j}-4 S
\end{array}\right]<0, \\
1 \leq i \leq j \leq r .
\end{array}
$$

Therefore, according to the fact of $\mu_{i}(\theta(k)) \geq 0$ in (8), the inequality (30) and the equality (24), we can obtain $\Delta V<0$.
Define sets $\Omega_{\sigma}=\left\{y \in R^{2 n}: y^{T} \theta_{\sigma i j} y<0\right\}$; thus $\cup \Omega_{i}=R^{2 n} \backslash\{0\}$. Construct the sets $\widetilde{\Omega}_{1}=\Omega_{1}, \ldots, \widetilde{\Omega}_{\sigma}=$ $\Omega_{\sigma}-\bigcup_{i=1}^{\sigma-1} \widetilde{\Omega}_{i}, \ldots$.

Obviously, we have $\bigcup_{i=1}^{l} \widetilde{\Omega}_{i}=R^{2 n} \backslash\{0\}, \widetilde{\Omega}_{i} \cap \widetilde{\Omega}_{j}=\Phi, i \neq j$. Construct a switching law as follows:

$$
\sigma(x(k))=i, \quad \text { when } x(k) \in \widetilde{\Omega}_{i}, \sigma \in M .
$$

Thus, $\Delta V(x(k))+x^{T}(k) Q x(k)+u_{\sigma}^{T}(k) R u_{\sigma}(k) \leq \xi^{T}(k) \theta_{\sigma i j} \xi(k)$ $<0, \forall \xi(k) \neq 0$, where $\xi(k)=\left[\begin{array}{ll}x^{T}(k) & x^{T}(k-1)\end{array}\right]^{T}$.

Notice that $F^{T} F \leq I$. Applying Lemmas 6 and 7 to the inequality (29), we have

$$
\left[\begin{array}{ccccccc}
\Psi & \Lambda & \Pi & 0 & 0 & 0 & 0 \\
* & -2 P+2 S & 0 & 2 I & \left(k_{\sigma i}+k_{\sigma j}\right)^{T} & \left(E_{i} k_{\sigma j}\right)^{T} & \left(E_{j} k_{\sigma i}\right)^{T} \\
* & * & -2 S & 0 & 0 & -\left(E_{i} k_{\sigma j}\right)^{T} & \left(E_{j} k_{\sigma i}\right)^{T} \\
* & * & * & -2 Q^{-1} & 0 & 0 & 0 \\
* & * & * & * & -2 R^{-1} & 0 & 0 \\
* & * & * & * & * & -\varepsilon_{\sigma} I & 0 \\
* & * & * & * & * & * & -\varepsilon_{\sigma} I
\end{array}\right]<0
$$

where

$$
\begin{gathered}
\Psi=-2 P^{-1}+\varepsilon_{\sigma}\left(D_{i} D_{i}^{T}+D_{j} D_{j}^{T}\right), \\
\Lambda=\Phi_{i}+\Phi_{j}+\Gamma_{i 0} k_{\sigma j}+\Gamma_{j 0} k_{\sigma i}, \\
\Pi=\Gamma_{i 1} k_{\sigma j}+\Gamma_{j 1} k_{\sigma i} .
\end{gathered}
$$

By inequality (32) left-multiplied and right-multiplied by $\operatorname{diag}\left(\begin{array}{lllllll}I & P^{-1} & P^{-1} & I & I & I & I\end{array}\right)$ and defining new variables $X=$ $P^{-1}, Y_{\sigma i}=k_{\sigma i} X$, and $Z=X S X$, we have

$$
\left[\begin{array}{ccccccc}
L & U & V & 0 & 0 & 0 & 0 \\
* & -2 X+2 Z & 0 & 2 X & \left(Y_{\sigma i}+Y_{\sigma j}\right)^{T} & \left(E_{i} Y_{\sigma j}\right)^{T} & \left(E_{j} Y_{\sigma i}\right)^{T} \\
* & * & -2 Z & 0 & 0 & -\left(E_{i} Y_{\sigma j}\right)^{T} & \left(E_{j} Y_{\sigma i}\right)^{T} \\
* & * & * & -2 Q^{-1} & 0 & 0 & 0 \\
* & * & * & * & -2 R^{-1} & 0 & 0 \\
* & * & * & * & * & -\varepsilon_{\sigma} I & 0 \\
* & * & * & * & * & * & -\varepsilon_{\sigma} I
\end{array}\right]<0
$$


where

$$
\begin{gathered}
L=-2 X+\varepsilon_{\sigma}\left(D_{i} D_{i}^{T}+D_{j} D_{j}^{T}\right), \\
U=\Phi_{i}+\Phi_{j}+\Gamma_{i 0} Y_{\sigma j}+\Gamma_{j 0} Y_{\sigma i}, \\
V=\Gamma_{i 1} Y_{\sigma j}+\Gamma_{j 1} Y_{\sigma i} .
\end{gathered}
$$

Taking (28) into account, for all admissible uncertainties, we can infer that as follows:

$$
\Delta V(x(k)) \leq-x^{T}(k) Q x(k)-u_{\sigma}^{T}(k) R u_{\sigma}(k) .
$$

According to (18), we can infer that there exists at least one $\sigma$ such that (29) is established. Therefore, the closed-loop system (14) is asymptotically stable under the controller (11) and the switching law (31). Moreover, we have

$$
\begin{aligned}
& \Delta V<\sum_{i=1}^{r} \mu_{i}^{2} x^{T}(k)\left(\mathrm{Q}+K_{\sigma i}^{T} R K_{\sigma i}\right) x(k) \\
&+2 \sum_{i=1}^{r} \sum_{j>i}^{r} \mu_{i} \mu_{j} x^{T}(k) \\
& \quad \times\left(Q+\frac{\left(K_{\sigma i}+K_{\sigma j}\right)^{T}}{2} R \frac{\left(K_{\sigma i}+K_{\sigma j}\right)}{2}\right) x(k),
\end{aligned}
$$

thus

$$
\begin{aligned}
& \sum_{i=1}^{r} \mu_{i}^{2} x^{T}(k)\left(Q+K_{\sigma i}^{T} R K_{\sigma i}\right) x(k) \\
& +2 \sum_{i=1}^{r} \sum_{j>i}^{r} \mu_{i} \mu_{j} x^{T}(k) \\
& \quad \times\left(Q+\frac{\left(K_{\sigma i}+K_{\sigma j}\right)^{T}}{2} R \frac{\left(K_{\sigma i}+K_{\sigma j}\right)}{2}\right) x(k)<-\Delta V .
\end{aligned}
$$

The inequalities (38) are added up together in the case that $k$ is $0,1,2, \ldots, \infty$; we have

$$
\begin{aligned}
J=\sum_{k=0}^{\infty}\left[\sum_{i=1}^{r} \mu_{i}^{2} x^{T}(k)\left(Q+K_{\sigma i}^{T} R K_{\sigma i}\right) x(k)\right. \\
+2 \sum_{i=1}^{r} \sum_{j>i}^{r} \mu_{i} \mu_{j} x^{T}(k) \\
\left.\quad \times\left(Q+\frac{\left(K_{\sigma i}+K_{\sigma j}\right)^{T}}{2} R \frac{\left(K_{\sigma i}+K_{\sigma j}\right)}{2}\right) x(k)\right] \\
\leq x^{T}(0) P x(0)+x^{T}(-1) S x(-1) .
\end{aligned}
$$

Therefore, Theorem 8 is proved.

Theorem 9. Consider the uncertain nonlinear networked control systems (3) and the cost function (9). If there exist some nonpositive or nonnegative constants $\delta_{\sigma \lambda}(\sigma, \lambda=1,2, \ldots, l)$, a group of positive constants $\varepsilon_{\sigma}>0$, and positive-definite matrices $X_{\sigma}, Z_{\sigma}$, matrix $Y_{\sigma i}, \sigma=1, \ldots, l, i=1, \ldots, r$, such that the following matrix inequalities:

$$
\left[\begin{array}{ccccccc}
L & U & V & 0 & 0 & 0 & 0 \\
* & \Xi+\sum_{\lambda=1, \lambda \neq \sigma}^{l} \delta_{\sigma \lambda}\left(X_{\lambda}-X_{\sigma}\right) & 0 & 2 X & \left(Y_{\sigma i}+Y_{\sigma j}\right)^{T} & \left(E_{i} Y_{\sigma j}\right)^{T} & \left(E_{j} Y_{\sigma i}\right)^{T} \\
* & * & -2 Z & 0 & 0 & -\left(E_{i} Y_{\sigma j}\right)^{T} & -\left(E_{j} Y_{\sigma i}\right)^{T} \\
* & * & * & -2 Q^{-1} & 0 & 0 & 0 \\
* & * & * & * & -2 R^{-1} & 0 & 0 \\
* & * & * & * & * & -\varepsilon_{\sigma} I & 0 \\
* & * & * & * & * & * & -\varepsilon_{\sigma} I
\end{array}\right]<0, \quad 1 \leq i \leq j \leq r,
$$

then system (14) with the guaranteed cost controller (11) and the switching law $\sigma=\sigma(x(k))$ is globally asymptotically stable. The guaranteed cost function (9) satisfies the following bound:

$$
J \leq x^{T}(0) P_{\sigma} x(0)+x^{T}(-1) S_{\sigma} x(-1) .
$$

Proof. By the Lemmas 6 and 7, and $X_{\sigma}=P_{\sigma}^{-1}, Y_{\sigma i}=k_{\sigma i} X_{\sigma}$, $Z_{\sigma}=X_{\sigma} S_{\sigma} X_{\sigma}$, from (40), we have 


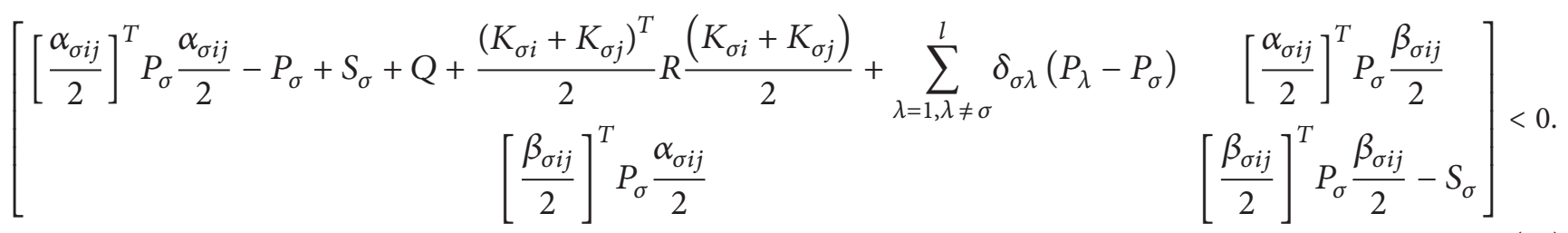

Without loss of generality, we assume that $\delta_{\sigma \lambda} \geq 0$.

Obviously, there exists at least one $\sigma \in M$ when $x(k) \in$ $R^{n} \backslash\{0\}$, such that $x^{T}(k)\left(P_{\lambda}-P_{\sigma}\right) x(k) \geq 0, \forall \lambda \in M$.

Let $\Omega_{\sigma}=\left\{x(k) \in R^{n} \mid x^{T}(k)\left(P_{\lambda}-P_{\sigma}\right) x(k) \geq 0, \forall \lambda \in\right.$ $M, \lambda \neq \sigma, x(k) \neq 0\}$; thus $\cup \Omega_{i}=R^{n} \backslash\{0\}$.

Construct the sets $\widetilde{\Omega}_{1}=\Omega_{1}, \ldots, \widetilde{\Omega}_{\sigma}=\Omega_{\sigma}-\bigcup_{i=1}^{\sigma-1} \widetilde{\Omega}_{i}, \ldots$.
Obviously, we have $\bigcup_{i=1}^{l} \widetilde{\Omega}_{i}=R^{n} \backslash\{0\}, \widetilde{\Omega}_{i} \cap \widetilde{\Omega}_{j}=\Phi, i \neq j$. Construct a switched law by

$$
\sigma(x(k))=i, \quad \text { when } x(k) \in \widetilde{\Omega}_{i}, \sigma \in M
$$

Thus, from (43), we have

$$
\begin{aligned}
& {\left[\begin{array}{c}
x(k) \\
x(k-1)
\end{array}\right]^{T}\left[\begin{array}{cc}
{\left[\frac{\alpha_{\sigma i j}}{2}\right]^{T} P_{\sigma} \frac{\alpha_{\sigma i j}}{2}-P_{\sigma}+S_{\sigma}+Q+\frac{\left(K_{\sigma i}+K_{\sigma j}\right)^{T}}{2} R \frac{\left(K_{\sigma i}+K_{\sigma j}\right)}{2}} & {\left[\frac{\alpha_{\sigma i j}}{2}\right]^{T} P_{\sigma} \frac{\beta_{\sigma i j}}{2}} \\
{\left[\frac{\beta_{\sigma i j}}{2}\right]^{T} P_{\sigma} \frac{\alpha_{\sigma i j}}{2}} & {\left[\frac{\beta_{\sigma i j}}{2}\right]^{T} P_{\sigma} \frac{\beta_{\sigma i j}}{2}-S}
\end{array}\right]} \\
& {\left[\begin{array}{c}
x(k) \\
x(k-1)
\end{array}\right]<0, \quad 1 \leq i \leq j \leq r .}
\end{aligned}
$$

Following the similar lines as in the proof of Theorem 8, we have

$$
\theta_{\sigma i j}=\left[\begin{array}{rr}
{\left[\frac{\alpha_{\sigma i j}}{2}\right]^{T} P_{\sigma} \frac{\alpha_{\sigma i j}}{2}-P_{\sigma}+S_{\sigma}+Q+\frac{\left(K_{\sigma i}+K_{\sigma j}\right)^{T}}{2} R \frac{\left(K_{\sigma i}+K_{\sigma j}\right)}{2}} & {\left[\frac{\alpha_{\sigma i j}}{2}\right]^{T} P_{\sigma} \frac{\beta_{\sigma i j}}{2}} \\
{\left[\frac{\beta_{\sigma i j}}{2}\right]^{T} P_{\sigma} \frac{\alpha_{\sigma i j}}{2}} & \left.\left[\frac{\beta_{\sigma i j}}{2}\right]^{T} P_{\sigma} \frac{\beta_{\sigma i j}}{2}-S\right]<0, \\
1 \leq i \leq j \leq r, & 1 \leq 1 \leq 1 \leq 1
\end{array}\right.
$$

then, $\Delta V_{\sigma}(x(k))+x^{T}(k) Q x(k)+u_{\sigma}^{T}(k) R u_{\sigma}(k)<0$. Thus,

$$
\begin{aligned}
& \sum_{i=1}^{r} \mu_{i}^{2} x^{T}(k)\left(Q+K_{\sigma i}^{T} R K_{\sigma i}\right) x(k) \\
& \quad+2 \sum_{i=1}^{r} \sum_{j>i}^{r} \mu_{i} \mu_{j} x^{T}(k)\left(Q+\frac{\left(K_{\sigma i}+K_{\sigma j}\right)^{T}}{2} R \frac{\left(K_{\sigma i}+K_{\sigma j}\right)}{2}\right) x(k) \\
& <-\Delta V_{\sigma} .
\end{aligned}
$$

The above inequality (47) is added up together in the case that $k$ is $0,1,2, \ldots, \infty$, according to the stability of the system, then

$$
J \leq x^{T}(0) P_{\sigma} x(0)+x^{T}(-1) S_{\sigma} x(-1) .
$$

Theorem 9 is proved.

\section{Simulation Example}

Consider the nonlinear system with the following differential equation [24]:

$$
\ddot{s}(t)+f(s(t), \dot{s}(t))-0.1 s(t)=F(t),
$$

where $f(s(t), \dot{s}(t))=0.5 s(t)+0.75 \sin (\dot{s}(t) / 0.5)$. 
Choose the state variable and the input variable as $x(t)=$ $[s(t), \dot{s}(t)]^{T}, u(t)=F(t)$, respectively. It can be represented by the following fuzzy model consisting of two rules:

$$
\begin{aligned}
R^{1}: & \text { IF } x_{2}(t) \text { is } M_{1}, \\
& \text { THEN } \dot{x}(t)=A_{1} x(t)+B_{1} u(t), \\
R^{2}: & \text { IF } x_{2}(t) \text { is } M_{2}, \\
& \text { THEN } \dot{x}(t)=A_{2} x(t)+B_{2} u(t),
\end{aligned}
$$

where $x(t)=\left[x_{1}(t), x_{2}(t)\right]^{T}$

$$
\begin{aligned}
& A_{1}=\left[\begin{array}{cc}
0 & 1 \\
0.1 & -2
\end{array}\right], \quad A_{2}=\left[\begin{array}{cc}
0 & 1 \\
0.1 & -0.5-1.5 \beta
\end{array}\right], \\
& B_{1}=B_{2}=\left[\begin{array}{ll}
-0.1 & -0.2
\end{array}\right]^{T}, \quad \beta=\frac{0.01}{\pi},
\end{aligned}
$$

and $\beta$ is used to avoid system matrices being singular.

The sampling period $T=0.3 \mathrm{~s}$, then the discrete model of the system is as follows:

$R^{1}: \operatorname{IF} \frac{x_{2}(t)}{0.5}$ is about 0,

$$
\begin{aligned}
\text { THEN } x(k+1)= & \Phi_{1} x(k)+\left(\Gamma_{10}+D_{1} F_{1}\left(\tau_{k}\right) E_{1}\right) u(k) \\
& +\left(\Gamma_{11}-D_{1} F_{1}\left(\tau_{k}\right) E_{1}\right) u(k-1),
\end{aligned}
$$

$R^{2}: \operatorname{IF} \frac{x_{2}(t)}{0.5}$ is about $\pi$ or $-\pi$,

$$
\begin{aligned}
\text { THEN } x(k+1)= & \Phi_{2} x(k)+\left(\Gamma_{20}+D_{2} F_{2}\left(\tau_{k}\right) E_{2}\right) u(k) \\
& +\left(\Gamma_{21}-D_{2} F_{2}\left(\tau_{k}\right) E_{2}\right) u(k-1),
\end{aligned}
$$

where

$$
\begin{aligned}
& \Phi_{1}=\left[\begin{array}{ll}
1.0037 & 0.2259 \\
0.0226 & 0.5519
\end{array}\right], \quad \Phi_{2}=\left[\begin{array}{ll}
1.0043 & 0.2788 \\
0.0279 & 0.8635
\end{array}\right], \\
& \Gamma_{10}=\left[\begin{array}{c}
-10.0018 \\
-0.0001
\end{array}\right], \quad \Gamma_{11}=\left[\begin{array}{c}
-10.0390 \\
-0.2260
\end{array}\right] \text {, } \\
& \Gamma_{20}=\left[\begin{array}{c}
-10 \\
0
\end{array}\right], \quad \Gamma_{21}=\left[\begin{array}{c}
-10.0432 \\
-0.2788
\end{array}\right] \text {, } \\
& D_{1}=\left[\begin{array}{cc}
20.7693 & 0.2141 \\
1.0137 & -0.4386
\end{array}\right] \text {, } \\
& D_{2}=\left[\begin{array}{cc}
6.8691 & 0.6676 \\
0.3353 & -1.3678
\end{array}\right] \text {, } \\
& E_{1}=\left[\begin{array}{l}
0.4773 \\
1.0869
\end{array}\right], \quad E_{2}=\left[\begin{array}{l}
1.2500 \\
1.4786
\end{array}\right] \text {, } \\
& F_{1}\left(\tau_{k}\right)=\left[\begin{array}{cc}
e^{-0.0488 \tau_{k}} & 0 \\
0 & e^{-2.0488\left(0.3-\tau_{k}\right)}
\end{array}\right] \text {, } \\
& F_{2}\left(\tau_{k}\right)=\left[\begin{array}{cc}
e^{-0.1522 \tau_{k}} & 0 \\
0 & e^{-0.6570\left(0.3-\tau_{k}\right)}
\end{array}\right] \text {. }
\end{aligned}
$$

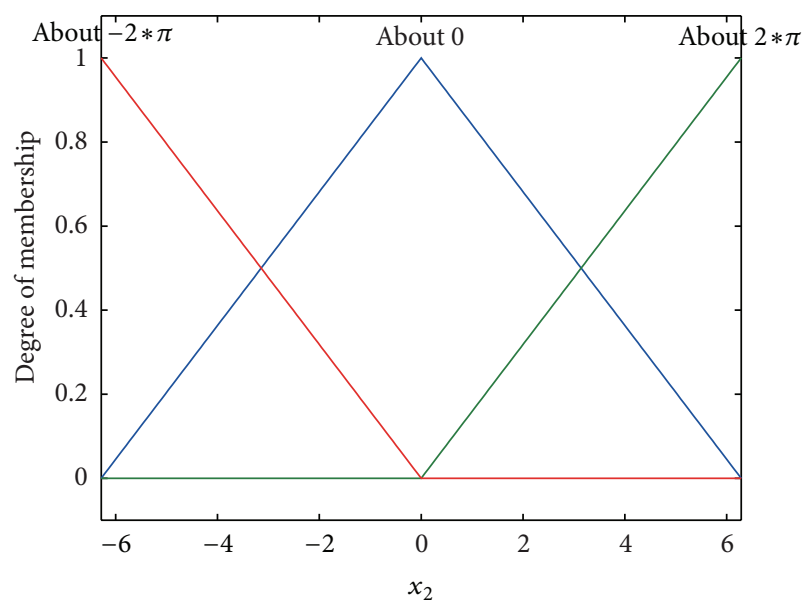

Figure 2: The membership function of input $x_{2}$.

Obviously, $F_{1}\left(\tau_{k}\right)$ and $F_{2}\left(\tau_{k}\right)$ satisfy the uncertain matching conditions:

$$
F_{1}^{T}\left(\tau_{k}\right) F_{1}\left(\tau_{k}\right) \leq I, \quad F_{2}^{T}\left(\tau_{k}\right) F_{2}\left(\tau_{k}\right) \leq I .
$$

The membership functions of "about 0 " and "about $\pi$ or $-\pi$ " are selected as in Figure 2.

Suppose the switched fuzzy feed-back controllers are the following fuzzy controllers:

$$
\begin{aligned}
& u_{1}(k)=\sum_{i=1}^{2} \mu_{i}\left(x_{2}(k)\right) k_{1 i} x_{i}(k), \\
& u_{2}(k)=\sum_{i=1}^{2} \mu_{i}\left(x_{2}(k)\right) k_{2 i} x_{i}(k) .
\end{aligned}
$$

Choose $Q=0.1 I_{2 \times 2}, R=1$. Carrying out computations for matrices inequality (40), we obtain

$$
\begin{aligned}
& P_{1}=\left[\begin{array}{ll}
8.0177 & 5.2362 \\
5.2362 & 8.5369
\end{array}\right], \\
& P_{2}=\left[\begin{array}{ll}
7.8926 & 5.4026 \\
5.4026 & 9.1487
\end{array}\right] .
\end{aligned}
$$

The controller gain:

$$
\begin{aligned}
& k_{11}=\left[\begin{array}{ll}
-0.6138 & -0.4204
\end{array}\right], \\
& k_{12}=\left[\begin{array}{ll}
-0.2936, & -0.3036
\end{array}\right], \\
& k_{21}=\left[\begin{array}{ll}
-0.4156 & -0.3852
\end{array}\right], \\
& k_{22}=\left[\begin{array}{ll}
-0.0893, & -0.1025]
\end{array} .\right.
\end{aligned}
$$

Let

$$
\begin{aligned}
& \Omega_{1}=\left\{x(k) \in R^{2} \mid x^{T}(k)\left(P_{2}-P_{1}\right) x(k) \geq 0, x(k) \neq 0\right\}, \\
& \Omega_{2}=\left\{x(k) \in R^{2} \mid x^{T}(k)\left(P_{1}-P_{2}\right) x(k) \geq 0, x(k) \neq 0\right\} .
\end{aligned}
$$




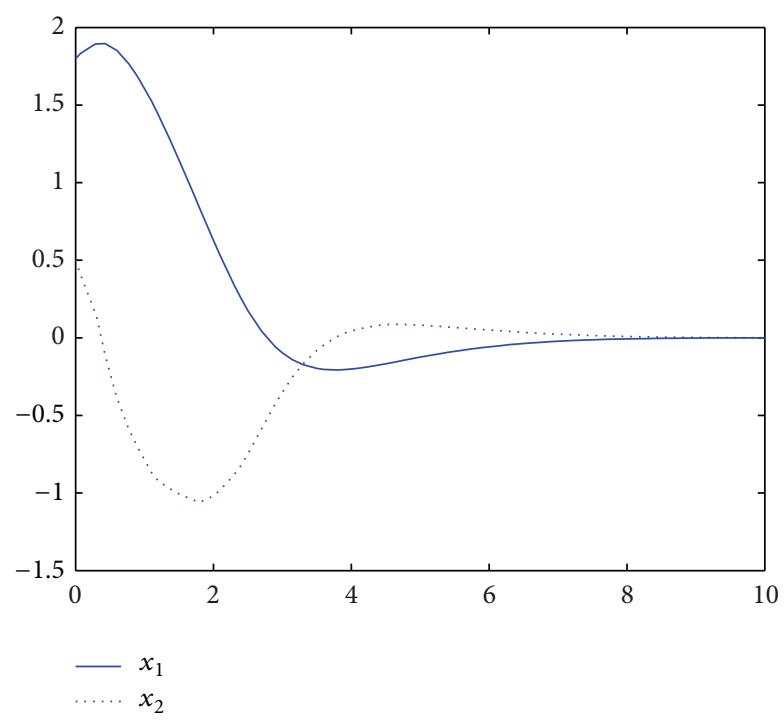

FIGURE 3: The state trajectory using fuzzy controller 1.

Then $\Omega_{1} \cup \Omega_{2}=R^{2} \backslash\{0\}$. We design a switched law as follows:

$$
\sigma(x(k))= \begin{cases}1, & x(k) \in \Omega_{1}, \\ 2, & x(k) \in \Omega_{2} \backslash \Omega_{1} .\end{cases}
$$

The initial condition is $\left[\begin{array}{ll}1.8 & 0.5\end{array}\right]^{T}$, guaranteed cost bound $J^{*}=36.53$.

Figures 3 and 4 show the system state trajectories that use fuzzy controller 1 and fuzzy controller 2, respectively. Figure 5 shows the simulating results for the proposed switched fuzzy controller method. In Figure 5, the system state trajectories indicate that nonlinear networked control system is asymptotically stable and satisfies the performance index via the designed guaranteed cost controller and the switching law. From the simulating results, we can confirm that the guaranteed cost controller in the switched fuzzy model is able to stabilize the nonlinear delay system via switching T$S$ fuzzy method. In addition, the performance of switching fuzzy controller is better than that of the fuzzy controllers.

\section{Conclusions}

In this paper, we have presented a novel controller design methodology for a class of nonlinear NCSs based on switched T-S fuzzy model. By introducing the switching mechanism into the fuzzy T-S systems, the proposed methods can deal with the uncertainties of nonlinear NCSs with time delays and furthermore avoid the inherent drawback of a fuzzy T-S model in controller design and implementation of nonlinear systems. In addition, considering QoS of nonlinear NCSs, some sufficient conditions for the existence of the robust guaranteed cost control law have been built via Lyapunov functional approach. Simulation results have verified and confirmed the effectiveness of the guaranteed cost controller based on the switched T-S fuzzy model for nonlinear NCSs.

At present, this paper only presents a numerical example to show the validity of our control scheme on the nonlinear

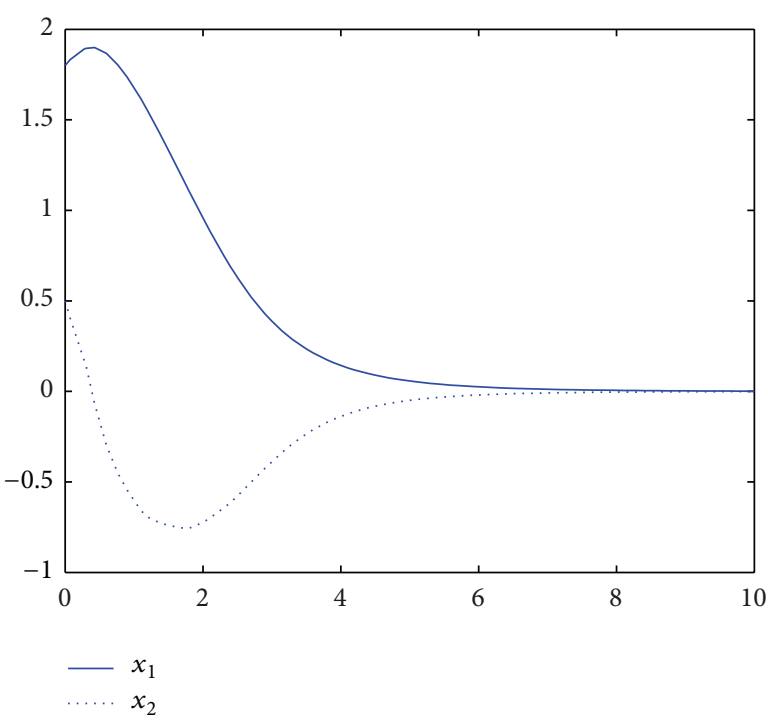

FIGURE 4: The state trajectory using fuzzy controller 2.

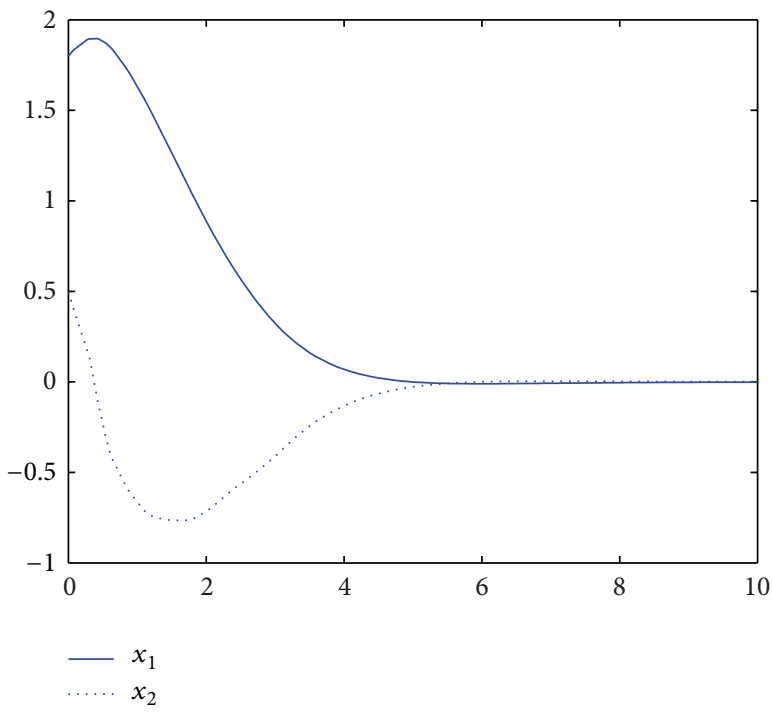

FIGURE 5: The state trajectory using switched fuzzy controller.

NCS with time delays. In next step, we plan to further verify this control scheme via practical NCSs and investigate the stability analysis and controller design with multiple-packet transmission in nonlinear NCSs. Moreover, the boundedness of the parameter constraints for NCSs will be studied. The switched dynamics of nonlinear NCSs will also be considered in future investigation.

\section{Conflict of Interests}

The authors declare that there is no conflict of interests regarding the publication of this paper. 


\section{Acknowledgments}

This work is supported by National Natural Science Foundation of China (Grant no. 50804061) and Scientific and Technological Research Program of Chongqing Municipal Education Commission (Grant no. KJ130522).

\section{References}

[1] D. Yue, Q.-L. Han, and C. Peng, "State feedback controller design of networked control systems," IEEE Transactions on Circuits and Systems II, vol. 51, no. 11, pp. 640-644, 2004.

[2] H. Zhang, D. Yang, and T. Chai, "Guaranteed cost networked control for T-S fuzzy systems with time delays," IEEE Systems, Man, and Cybernetics Society, vol. 37, no. 2, pp. 160-172, 2007.

[3] X. Luan, P. Shi, and F. Liu, "Stabilization of networked control systems with random delays," IEEE Transactions on Industrial Electronics, vol. 58, no. 9, pp. 4323-4330, 2011.

[4] B. Rahmani and A. H. D. Markazi, "Variable selective control method for networked control systems," IEEE Transactions on Control Systems Technology, vol. 21, no. 3, pp. 975-982, 2012.

[5] D. Nešić and A. R. Teel, "Input-to-state stability of networked control systems," Automatica, vol. 40, no. 12, pp. 2121-2128, 2004.

[6] H. Chu, S. Fei, D. Yue, C. Peng, and J. Sun, " $H_{\infty}$ quantized control for nonlinear networked control systems," Fuzzy Sets and Systems, vol. 174, pp. 99-113, 2011.

[7] J. Gao, H. Su, X. Ji, and J. Chu, "Robust stabilization for a class of nonlinear networked control systems," Journal of Control Theory and Applications, vol. 6, no. 3, pp. 300-304, 2008.

[8] H. Xu and S. Jagannathan, "Stochastic optimal controller design for uncertain nonlinear networked control system neuro dynamic programming," IEEE Transactions On Neural Networks And Learning Systems, vol. 24, no. 3, pp. 471-484, 2013.

[9] T. Takagi and M. Sugeno, "Fuzzy identification of systems and its applications to modeling and control," IEEE Transactions on Systems, Man and Cybernetics, vol. 15, no. 1, pp. 116-132, 1985.

[10] R.-J. Wang, W.-W. Lin, and W.-J. Wang, "Stabilizability of linear quadratic state feedback for uncertain fuzzy time-delay systems," IEEE Transactions on Systems, Man, and Cybernetics B, vol. 34, no. 2, pp. 1288-1292, 2004.

[11] B. Chen and X. Liu, "Fuzzy guaranteed cost control for nonlinear systems with time-varying delay," IEEE Transactions on Fuzzy Systems, vol. 13, no. 2, pp. 238-249, 2005.

[12] X.-P. Guan and C.-L. Chen, "Delay-dependent guaranteed cost control for T-S fuzzy systems with time delays," IEEE Transactions on Fuzzy Systems, vol. 12, no. 2, pp. 236-249, 2004.

[13] C. Hua and S. X. Ding, "Decentralized networked control system design using T-S fuzzy approach," IEEE Transactions on Fuzzy Systems, vol. 20, no. 1, pp. 9-21, 2012.

[14] F. Delmotte, T. M. Guerra, and M. Ksantini, "Continuous Takagi-Sugeno's models: reduction of the number of LMI conditions in various fuzzy control design technics," IEEE Transactions on Fuzzy Systems, vol. 15, no. 3, pp. 426-438, 2007.

[15] D. Jabri, K. Guelton, N. Manamanni et al., "Robust stabilization of nonlinear systems based on a switched fuzzy control law," Control Engineering And Applied Informatics, vol. 14, no. 2, pp. 40-49, 2012.

[16] H. Ohtake, K. Tanaka, and H. O. Wang, "Switching fuzzy controller design based on switching lyapunov function for a class of nonlinear systems," IEEE Transactions on Systems, Man, and Cybernetics B, vol. 36, no. 1, pp. 13-23, 2006.

[17] H. K. Lam, "Stability analysis of sampled-data fuzzy controller for nonlinear systems based on switching T-S fuzzy model," Nonlinear Analysis, vol. 3, no. 4, pp. 418-432, 2009.

[18] H. Yang and J. Zhao, "Robust control for a class of uncertain switched fuzzy systems," Journal of Control Theory and Applications, vol. 5, no. 2, pp. 184-188, 2007.

[19] B. Niu and J. Zhao, "Barrier Lyapunov functions for the output tracking control of constrained nonlinear switched systems," Systems \& Control Letters, vol. 62, no. 10, pp. 963-971, 2013.

[20] H. K. Lam, F. H. F. Leung, and Y. S. Lee, "Design of a Switching Controller for Nonlinear Systems With Unknown Parameters Based on a Fuzzy Logic Approach," IEEE Transactions on Systems, Man, and Cybernetics B, vol. 34, no. 2, pp. 1068-1074, 2004.

[21] G.-H. Yang and J. Dong, "Switching fuzzy dynamic output feedback $\mathrm{H} \infty$ control for nonlinear systems," IEEE Transactions on Systems, Man, and Cybernetics B, vol. 40, no. 2, pp. 505-516, 2010.

[22] Y. Sun and J. Xu, "Stability analysis and controller design for networked control systems with random time delay," in Proceedings of the 9th International Conference on Electronic Measurement and Instruments (ICEMI '09), pp. 136-141, August 2009.

[23] L. Xie, "Output feedback $H_{\infty}$ control of systems with parameter uncertainty," International Journal of Control, vol. 63, no. 4, pp. 741-750, 1996.

[24] X.-P. Guan and C.-L. Chen, "Delay-dependent guaranteed cost control for T-S fuzzy systems with time delays," IEEE Transactions on Fuzzy Systems, vol. 12, no. 2, pp. 236-249, 2004. 


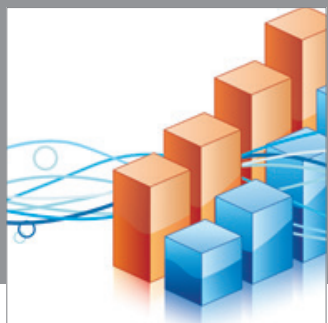

Advances in

Operations Research

mansans

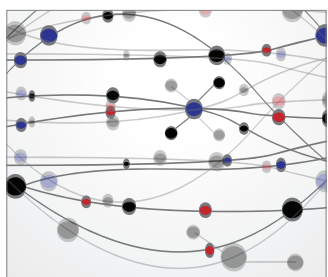

The Scientific World Journal
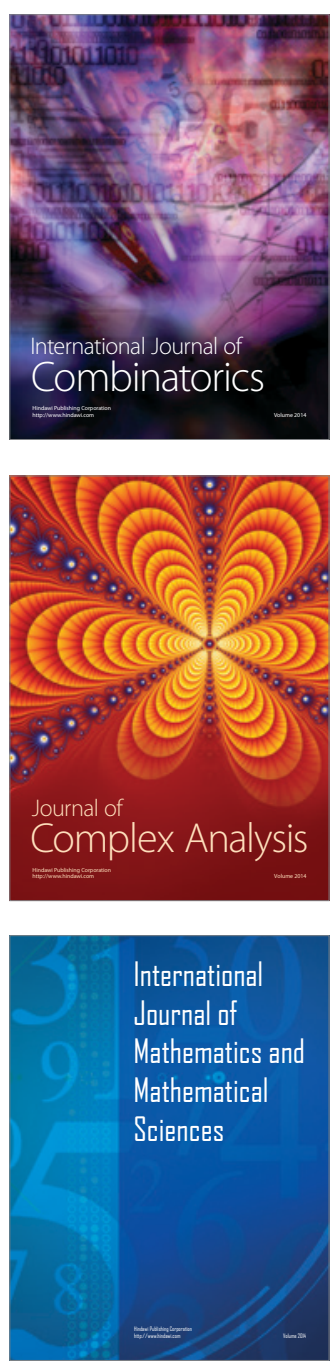
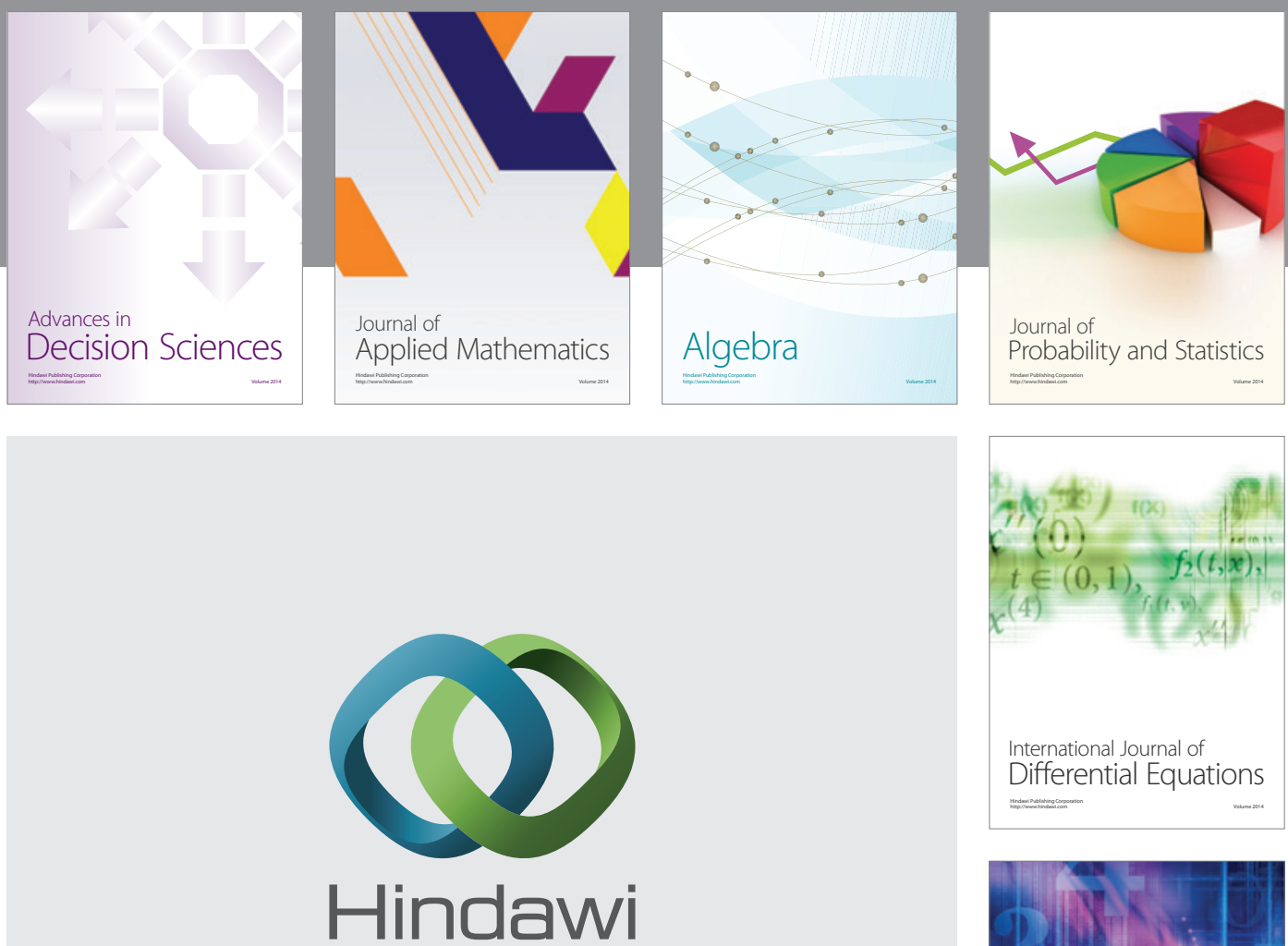

Submit your manuscripts at http://www.hindawi.com
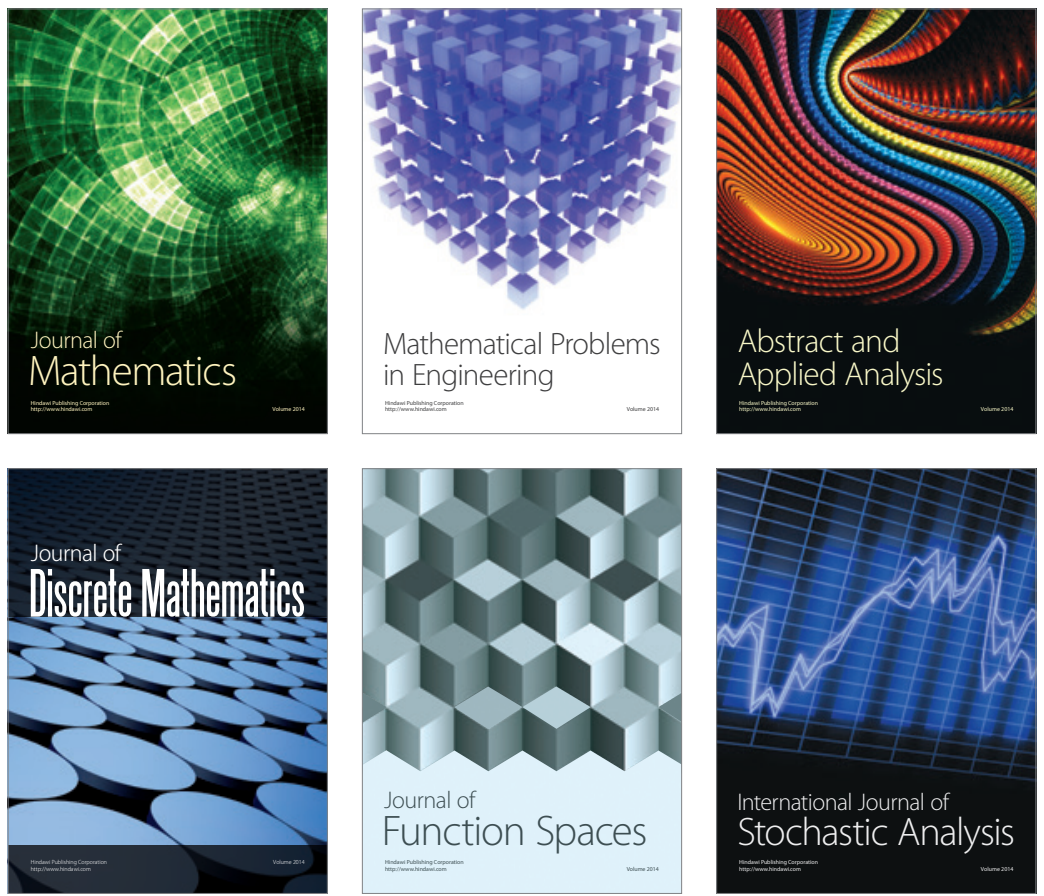

Journal of

Function Spaces

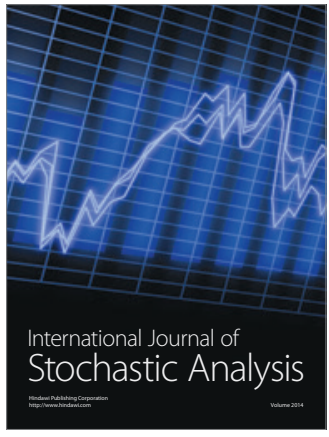

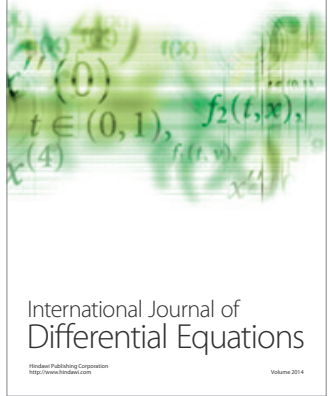
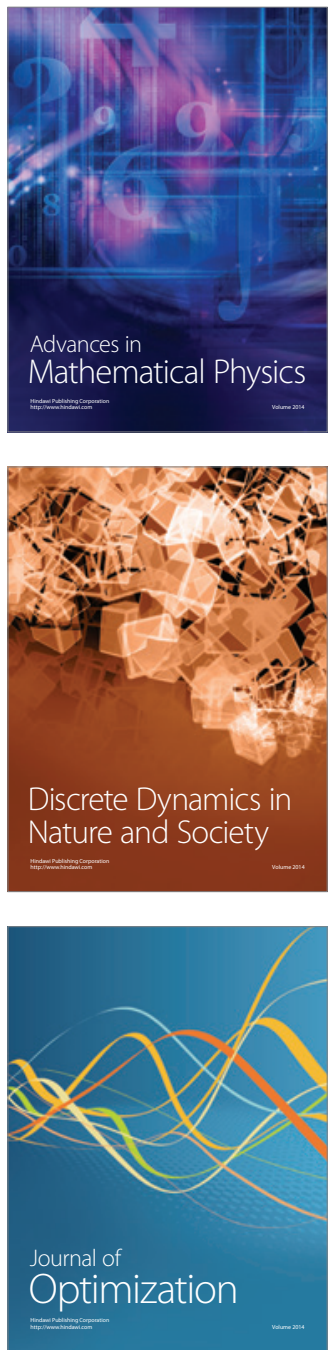